Note on Dimensions of Physical Quantities

This content has been downloaded from IOPscience. Please scroll down to see the full text. 1903 Proc. Phys. Soc. London 18445

(http://iopscience.iop.org/1478-7814/18/1/341)

View the table of contents for this issue, or go to the journal homepage for more

Download details:

IP Address: 128.119.168.112

This content was downloaded on 01/10/2015 at 12:36

Please note that terms and conditions apply. 
In condusion, I desire to acknowledge that I have had the advantage of discussing with my friend $\mathrm{Mr}$. Sowter the subjects of this paper, in the preparation of which ho has taken great interest.

\section{Note on Dimensions of Physical Quantities.} $B y$ R. J. Sowter, B.Sc., A.R.C.Sc.*

Mr. RaveNSHEAR in his paper on dimensional analysis of physical quantities has shown that any physical quantity, $Q$ say, is expressible in terms of the dynamical quantities, $L$, $M$, and $T$, in different ways, but that all the various ways are related to or connected with one another by an index law. Thus :-

$$
\begin{aligned}
& Q=\mu^{a}\left(\mathrm{~L}^{a} \mathrm{M}^{\beta} \mathrm{T}^{\gamma}\right)=\kappa^{b}\left(\mathrm{~L}^{a^{\prime}} \mathrm{M}^{\beta^{\prime}} \mathrm{T}^{\prime}\right)=\gamma^{c}\left(\mathrm{~L}^{\alpha^{\prime \prime}} \mathrm{M}^{\beta^{\prime}} \mathrm{T}^{\prime \prime}\right)=q^{d}\left(\mathrm{D}^{n}\right), \\
& \alpha+\beta+\gamma=\alpha^{\prime}+\beta^{\prime}+\gamma^{\prime}=\alpha^{\prime \prime}+\beta^{\prime \prime}+\gamma^{\prime \prime}=n .
\end{aligned}
$$

Now this index law not only affords a useful working check in dimensional analysis, but in my opinion it appears to be pregnant with suggestion, and calls for careful consideration. It is obvious that one interpretation is that the dynamical factors are complete in themselves; that is, $\mu^{a}, \kappa^{b}, \gamma^{c}, q^{d}$ do not contain dynamical factors, or are not rationalizing factors in a dynamical sense, and that these factors show the various ways in which quantitative measurements of the physical quantity $Q$ are made. The dynamical factors consequently express change-ratios, and have no qualitative significanco. The things $Q$ and $\left(L^{\alpha} M^{\beta} T^{\gamma}\right)$ are then related in their quantitative measurements merely, they are not identifiablo qualitatively. This view seems to me to be logically complete in itself, and is probably the aspect of dimensional analysis conceived by Fourier.

The other interpretation, namely, that $\left(L^{\alpha} M^{\beta} T^{\gamma}\right) \ldots\left(D^{n}\right)$ are stanped with qualitative significance requires the factors $\mu, \kappa$, \&c. to be dynamically related, and does not require that any index law should hold. It is immaterial with this view whether $(\alpha+\beta+\gamma)$ is or is not equal to $\left(x^{\prime}+\beta^{\prime}+\gamma^{\prime}\right)$, the only

$$
\text { * Read April 24, } 1003 .
$$


condition necessary is that

$$
\frac{\mu^{a}}{\kappa^{b}}=\frac{L^{a^{\prime}} M^{\beta^{\prime}} T^{\gamma}}{L^{a} M^{\beta} T^{\gamma}}
$$

Now this view requires all physical quantities to be expressible completely in dynamical terms, that is, Laplace's doctrine of a dynamical universe must be accepted. Advances are being made in this direction; but the logical position of this complete dyuamical resolution of the physical universe, it must be admitted, is not,more forward than in the time of Laplace. This interpretation is by no means logically complete.

That view which relates all things capable of quantitative measurement through quantity alone seems to be thrust upon us by the facts, although the whole endeavour and trend of modern physics is to explain things dynamically. The factors $\mu^{a}, \kappa^{b}$, \&c., that we have been compelled to introduce into the various branches of physics carry with them the physical qualities or characteristics of the quantities associated with them.

The elimination of physical significance from any dynanical factor in the expression for a pbysical quantity does not appear to lead to failure along any line of argument. Further, elimination of physical significance can be applied to dynamical quantities themselves; so that, accepting this hypothesis, physical quantities, including dynamical quantities, can all be conceived to have a measuring dynamical factor. For example, (Velocity) $v=\mathrm{A}\left(\frac{\mathrm{L}}{\mathrm{T}}\right)$ would be looked on as measured by $L / T$, but to have the quality of $A$. That is, $A$ is here a velocity.

Also, if we consider Electric quantity Q,

$$
\mathrm{Q}=z^{-1} \mathrm{M}=\kappa^{\frac{1}{2}}\left(\mathrm{M}^{\frac{1}{2}} \mathrm{~L}^{\frac{3}{2}} \mathrm{~T}^{-1}\right)=\mu^{-\frac{1}{2}}\left(\mathrm{M}^{\frac{1}{2}} \mathrm{~L}^{\frac{1}{2}}\right),
$$

and rols the dynamical factors of physical significance, then

$$
(Q)=\left(z^{-1}\right)=\left(\kappa^{\frac{1}{2}}\right)=\left(\mu^{-\frac{1}{2}}\right) \text {, }
$$

that is, $Q$ is physically like $\frac{1}{z}, \sqrt{\kappa}$, and $\frac{1}{\sqrt{\mu}}$; and $\frac{1}{z}, \sqrt{\kappa}$, $\frac{1}{\sqrt{\mu}}$ are all physically a ilio or qualitatively sinilar. These 
factors must, on this hypothesis, be conceived as associated, say as wether factors.

Any physical quantity, on this hypothesis, is expressible as

$$
\mathrm{Q}=\mathrm{N}\left(\mathrm{D}^{n}\right) \gamma,
$$

where $\mathrm{N}$ is a nere number, $\left(D^{n}\right)$ is a dynamical factor indicating a quantitative measurement process, and $q$ is a quality factor of the nature of $Q$.

\section{Discussion ON THE TWO FOREgOING PAPERS.}

Prof. Everetr said that the possibility of making two fundamental units suffice instead of three, by taking advantage of the law of gravitational attraction, was nothing new, but uas expounded and worked out in some of its applications in ordinary text-books. The proposal to diminish the number of fundamental units by making the unit of length proportional to the unit time, simply amounted to the adoption of a fundamental unit of velocity to take the place of a unit length, and was no simplification. The supposed new law of indices appeared to be simply the well-known principle by which information as to physical relations could often be deduced from consideration of dimensions: by which, for example, it could be shown that the velocity of sound must vary directly as the square root of elasticity and inversely as the square root of density.

Mr. PRICE referred to the fact that the author bad detertermined the dimensions of mass in terms of length and time from the equation $f=m^{2} / l^{2}$, considering $G$, the Newtoninn constant of gravitation, as a quantity without dimensions. He thought that if all suppressed dimensions were properly introuluced and proper attention paid to the direction of vector quantities, then the dimensions of a quantity gave an idea of its nature, and quantities having the same dimensions were identical in their natures.

Prof. S. P. Thompson said he had read the paper through, and thought it brought the subject no further forward. There were several statements which were so surprising that it was necessury to give them consideration. For instance, we were asker to believe that a scalar, like mass, was of the same dimensions and nature as a vector, like length, and that 
both of these were physically the same as time. There is no distinction at all in the paper between scalars and vectors. The author's criterion for an undimensional quantity was a false one, and led to obviously incorrect results. Taking the formula $\mathrm{F}=\mathrm{M} a$, he (Prof. Thompson) said that, using the author's criterion, mass had no dimensions. The criterion also made $G$, the Newtonian constant, an undimensional quantity. Referring to the equation $\mathrm{Q}=\mathrm{N}\left(\mathrm{D}^{n}\right) q$ given in Mr. Sowter's Note, he thought that the right-hand side might contain a fourth letter representing a geometrical operator. Mr. Ravenshear in his paper had retained fractional exponents which were meaningless and could not possibly exist.

Mr. RAVENSHEAR said that much of the criticism appeared to him to be based upon misunderstandings of his position and the propositions he had advanced. He did not think that $G$ necessarily had no dimensions. His contention was that, owing to its universal constancy, to give it dimensions was not logically compulsory, and therefore it was optional to work out the consequences of both alternatives. He would have preferred that Prof. Thompson had criticized his criterion using Joule's equivalent, the example dealt with in his paper. The example used by Prof. Thompson did not satisfy the criterion, since masses might have any magnitude. He had not advocated the retention of irrational indices, but had put in a plea for two or three systems in which all the indices were integral. Prof. Everett had discussed the matter as though a new system of units was being proposed for practical adoption. Mr. Ravenshear said that, on the contrary, his object was merely the logical analysis of the principles underlying the use of units. He had not proposed to use velocity in place of length or time, but contended that a permanent motion furnished in a single standard two units from which all other dynamical units could in principle be derived.

Mr. Sowter said that Mr. Ravenshear had shown an alternative method of rationalizing irrational expressions. Referring to Prof. Thompson's observation that the equation $\mathrm{Q}=\mathbf{N}\left(\mathbf{D}^{n}\right) y$ required a further term representing a geometrical operator, he pointed out that if different directions 
are accounted for by symbols, these take their places in the dynamical or measuring factors $\left(\mathrm{D}^{n}\right)$, so that any physical quantity need only be expressed by the three factors set forth. As regards Prof. Everett's remark on the index law, he was aware of the application of dimensional analysis to the solution of certain physical problems, but he was not aware that the indox law had heen formulated and the deductions based upon it dealt with previously.

XLIT. On the Comparison of Vapour-Temperatures at Equal Pressures. $\quad$ By Professor J. D. Everett, F.R.S.

RAMSAX and Young seem to have been the first to call attention (Phil. Mag. Jar. 1886) to the fact that the ratio $t / t^{\prime}$ of the absolute temperatures at which two vapours (at saturation) have the same pressure $p$ remains nearly constant for changes of $p$ of very considerable magnitude. In tho case of vapours of kindred constitution, their results show that a twentyfold increase of $p$ only changes $t / t^{\prime}$ by about $\frac{1}{2}$ per cent.

They further lay down, for the comparison of vapours generally, the law-now known as "Ramsay and Young's law"-that if $t_{1}, t_{2}$ denote the absolute temperatures of one vapour at the pressures $p_{1} p_{2}$, and $t_{1}^{\prime} t_{2}{ }^{\prime}$ those of another vapour at the same pressures, we shall have

$$
\frac{t_{2}}{t_{2}^{\prime}}-\frac{t_{1}}{t_{1}^{\prime}}=c\left(t_{2}-t_{1}\right), \quad \text {. . . . . }
$$

$c$ being a small positive or negative constant multiplior, depending on the substances compared.

To the eye of the mathematician there is an awkward one-sidedness about this formula; it is not symmetrical as between $t$ and $t^{\prime}$. It can, however, be rendered symmetrical by first writing it in the form

$$
\frac{t}{t^{\prime}}-c t=k,
$$

